\title{
Myeloid Neoplasms with Germline Predisposition Associated with Telomerase Biology Disorders
}

National Cancer Institute

\section{Source}

National Cancer Institute. Myeloid Neoplasms with Germline Predisposition Associated

with Telomerase Biology Disorders. NCI Thesaurus. Code C151921.

Familial myelodysplastic syndromes/ acute myeloid leukemias associated with

telomerase biology disorders. 